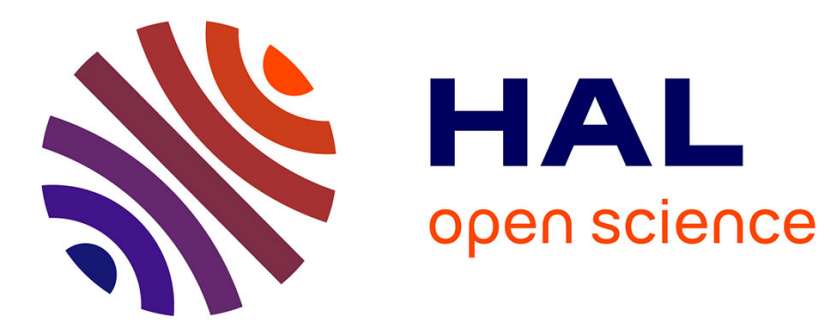

\title{
Splitting of a turbulent puff in pipe flow
}

Masaki Shimizu, Paul Manneville, Yohann Duguet, Genta Kawahara

\section{To cite this version:}

Masaki Shimizu, Paul Manneville, Yohann Duguet, Genta Kawahara. Splitting of a turbulent puff in pipe flow. Fluid Dynamics Research, 2014, 46, pp.61403. 10.1088/0169-5983/46/6/061403 . hal01102510

\section{HAL Id: hal-01102510 \\ https://hal-polytechnique.archives-ouvertes.fr/hal-01102510}

Submitted on 12 Jan 2015

HAL is a multi-disciplinary open access archive for the deposit and dissemination of scientific research documents, whether they are published or not. The documents may come from teaching and research institutions in France or abroad, or from public or private research centers.
L'archive ouverte pluridisciplinaire HAL, est destinée au dépôt et à la diffusion de documents scientifiques de niveau recherche, publiés ou non, émanant des établissements d'enseignement et de recherche français ou étrangers, des laboratoires publics ou privés. 


\title{
Splitting of a turbulent puff in pipe flow
}

\author{
Masaki Shimizu ${ }^{1}$, Paul Manneville ${ }^{2}$, Yohann Duguet ${ }^{3}$ and \\ Genta Kawahara ${ }^{1}$ \\ ${ }^{1}$ Graduate School of Engineering Science, Osaka University, Toyonaka, 560-8531, \\ Japan \\ ${ }^{2}$ LadHyX, CNRS-UMR 7646, Ecole Polytechnique, F-91128, Palaiseau, France \\ ${ }^{3}$ LIMSI-CNRS, UPR 3251, Université Paris-Sud, F-91403 Orsay, France \\ E-mail: shimizu@me.es.osaka-u.ac.jp
}

\begin{abstract}
The transition to turbulence of the flow in a pipe of constant radius is numerically studied over a range of Reynolds numbers where turbulence begins to expand by puff splitting. We first focus on the case Re $=2300$ where splitting occurs as discrete events. Around this value only long-lived pseudo-equilibrium puffs can be observed in practice, as typical splitting times become very long. When Re is further increased, the flow enters a more continuous puff splitting regime where turbulence spreads faster. Puff splitting presents itself as a two-step stochastic process. A splitting puff first emits a chaotic pseudopod made of azimuthally localized streaky structures at the downstream (leading) laminar-turbulent interface. This structure can later expand azimuthally as it detaches from the parent puff. Detachment results from a collapse of turbulence over the whole cross-section of the pipe. Once the process is achieved a new puff is born ahead. Large-deviation consequences of elementary stochastic processes at the scale of the streak are invoked to explain the statistical nature of splitting and the Poisson-like distributions of splitting times reported by Avila, Moxey, de Lozar, Avila, Barkley and Hof (2011 Science 333 192-196).
\end{abstract}

Keywords: Transition to turbulence, Circular pipe, Puffs 


\section{Introduction}

In 1883, Osborne Reynolds experimentally discovered that the state of the flow in a pipe depends on a single parameter, now called Reynolds number Re, and that it changes qualitatively from laminar to turbulent at some critical value about 2000, under sufficiently large disturbances (Reynolds 1883). As is now well recognized, he was confronted to the most complicated case of transition to turbulence, the subcritical one, that occurs when the base flow profile is free from inflection point and is typical of wallbounded flows. In contrast to inflectional shear flows that become turbulent in a more continuous supercritical way via inertial instabilities of Kelvin-Helmholtz type, wallbounded flows turn out to be possibly linearly unstable against Tollmien-Schlichting waves at high Reynolds numbers only. Pipe flow appears to be an extreme case where steady laminar pipe flow happens to be linearly stable for all Reynolds numbers (Salwen et al 1980). Plane Couette flow (PCF), the simple shear flow between counter-sliding plates, is another such example (Romanov 1973) to be evoked later. In these cases, finite amplitude perturbations are indeed needed to trigger the transition to turbulence. Accordingly, there exists a whole range of Reynolds numbers, called the transitional range, where laminar flow can coexist with turbulence, both in parameter space and in physical space, i.e. with localized turbulent domains separated from laminar flow by front-like interfaces. A global stability threshold $\mathrm{Re}_{\mathrm{g}}$ can be defined, below which laminar flow prevails in the long time limit whatever the amplitude and shape of the initial perturbation. The determination of this threshold turns out to be particularly difficult, due to the fact that, below it, these patches of localized turbulence may persist for very long times before they ultimately decay.

In pipe flow axially localized turbulent plugs (Reynolds 1883, Coles 1962), called puffs (Wygnanski and Champagne 1973, Wygnanski et al 1975), can be observed in the lowest part of the transitional range. Figure 1 displays such a state as obtained in our numerical simulations. When Re increases, weakly turbulent puffs become growing turbulent slugs filled with small scale turbulence of regularly increasing length tending to invade the pipe (Wygnanski and Champagne 1973, Duguet et al 2010). In contrast, the length of the puffs is statistically constant, which led to think that they were "equilibrium" states (Wygnanski et al 1975). Their structure and sustainment mechanism have been much studied. Laminar flow enters the puff upstream, through an abrupt trailing edge, and leaves it downstream, through a smoother leading edge (Bandyopadhyay 1986, Shimizu and Kida 2009, Duguet et al 2010, Holzner et al 2013).

Recent experimental and numerical studies have shown that, although puffs have long lifetimes, they may decay to laminar flow within a finite time with some probability function of Re, pointing to a super-exponential increase of the lifetime with Re (Hof et al 2008). As Re gets larger, the turbulent activity grows via an increase of the length of individual puffs, while their decay probability becomes vanishingly small. While trying to equilibrate at a longer length, the puffs may split (Lindgren 1959), 


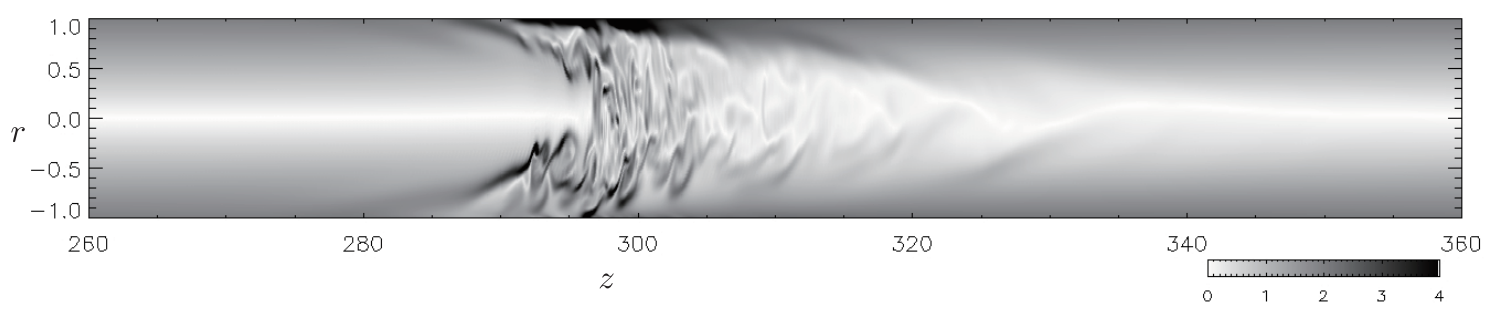

Figure 1. Pseudo-equilibrium puff at $R e=2000$. The absolute vorticity distribution in a plane through the pipe's centerline is represented using a linear gray scale. Only a part of the solution is displayed, the length of the computational domain being $L=400$.

progressively yielding trains of puffs (Nishi et al 2008, Avila et al 2011). As Re continues to increase, successive puffs turn into slugs with more abrupt leading edges (Wygnanski and Champagne 1973, Duguet et al 2010). Slugs tend to merge while the turbulent fraction - the fraction of the pipe occupied by developed turbulence - increases (Rotta 1956, Sreenivasan and Ramshankar 1986, Moxey and Barkley 2010, Avila and Hof 2013).

Puff splitting is a stochastic process with an exponential distribution of waiting times before splitting. The mean waiting time has been shown to decrease superexponentially as Re increases (Avila et al 2011). A competition between spontaneous decay of turbulence and propagation by splitting thus takes place, and a threshold can be defined when the mean waiting times for decay and splitting are equal, which was found for $\mathrm{Re}_{\mathrm{g}} \simeq 2040$ (Avila et al 2011). A simplified model accounting for the phenomenon has been devised by Barkley ((Barkley 2011b, Barkley 2011a)).

The present contribution is dedicated, not to the statistics of splitting, but to its local mechanics, i.e. a description of how the flow passes from one parent puff to a parent-and-child pair of puffs with some laminar gap between them, next to clusters of puffs at higher Re, and to a discussion of the statistical nature of the processes involved.

\section{Equations and numerical resolution}

We consider the flow of an incompressible viscous fluid driven by an external force imposing a time-independent bulk speed $U$ in a straight circular pipe of radius $a$. All physical quantities are non-dimensionalized by $a$ for length and the centerline speed $2 U$ of Hagen-Poiseuille flow for velocity. The Reynolds number is defined as Re $\equiv 2 a U / \nu$, $\nu$ being the kinematic viscosity. The continuity and Navier-Stokes equations governing the flow then read:

$$
\begin{aligned}
& \nabla \cdot \boldsymbol{u}=0, \\
& \frac{\partial \boldsymbol{u}}{\partial t}+(\boldsymbol{u} \cdot \nabla) \boldsymbol{u}=-\nabla p+\frac{1}{\operatorname{Re}} \nabla^{2} \boldsymbol{u}
\end{aligned}
$$

whare $\boldsymbol{u}$ and $p$ are velocity and pressure respectively. No-slip boundary conditions are imposed at the wall of the pipe and periodic conditions with period $L$ in the $z$ 
(streamwise) direction:

$$
\boldsymbol{u}(1, \theta, z)=0, \quad \boldsymbol{u}(r, \theta, z+L)=\boldsymbol{u}(r, \theta, z) .
$$

The reduced pressure $p$ includes an external force which is determined at each time to keep the bulk speed constant in time. The solenoidal field $\boldsymbol{u}$ can be expressed as

$$
\boldsymbol{u}=\nabla \times(\psi \hat{\boldsymbol{z}})+\nabla \times(\nabla \times(\phi \hat{\boldsymbol{z}})) .
$$

Using a spectral method, we numerically solved the set of evolution equations for $(\psi, \phi)$ straightforwardly derived from (1) and (2). These scalar functions were approximated by truncated expansions:

$$
(\psi, \phi)=\sum_{k=-K}^{K} \sum_{m=-M}^{M} \sum_{\substack{n=|m| \\ n+m=\text { even }}}^{N}\left(\hat{\psi}_{n}^{m k}, \hat{\phi}_{n}^{m k}\right) \Phi_{n}^{m}(r) \exp \mathrm{i}[m \theta+2 \pi k z / L]
$$

where $K, M$ and $N(\geq M)$ are positive integers and $\Phi_{n}^{m}(r)$ are Zernike circular polynomials. The time stepping algorithm combines a Crank-Nicholson scheme with a second order Adams-Bashforth scheme for the nonlinear terms, and the timestep is fixed to $\Delta t=0.005$; see (Shimizu and Kida 2008) for details. Here, a very long computational domain of length $L=400$ has been considered in order to reduce the influence of the periodic boundary conditions as much as possible. We have taken $(N, M, K)=(84,42,1535)$, which is thought to be well resolved. Preliminary work with $(N, M, K)=(40,21,1535)$ showed the same phenomenology, apart from a downward shift of Reynolds numbers relevant to the transitional range of about 200. A similar consequence of under-resolution has been reported for PCF (Manneville \& Rolland 2011).

\section{Splitting events}

Puff splitting has been studied by means of simulations all starting from single puff states at $R e=1900$ or 2000 . The Reynolds number was then set to its working value, from $\operatorname{Re}=2100$ to 2500 . Turbulent regions were unambiguously identified by using $\left|u_{z}-u^{\text {lam }}\right| \gtrsim 0.15$ as a criterion, $u_{z}$ and $u^{\text {lam }}$ being the perturbed and laminar axial speed measured at relevant distance from the centerline, $r=0$ in figure 2 , and $r=0.8$ in the other spatiotemporal diagrams, see below. Computations were continued as long as a significant laminar interval was preserved, modulo the axial periodic boundary conditions.

For $\operatorname{Re}=2100$ and 2200 (not illustrated) the mean splitting time is so long that no splitting could be expected, and indeed not detected, within observation times of order a few thousand time units. These results are consistent with Fig. 5 in Avila et al. (2011). Figure 2 shows the birth and growth of puffs at $R e=2300,2400$, and 2500. It displays spatiotemporal diagrams of the centerline streamwise velocity component in a reference frame moving with the bulk velocity $u_{\mathrm{m}}=\frac{1}{2} u_{\mathrm{cl}}=0.5$. These individual puffs have statistically well defined lengths as expected, the one at $\operatorname{Re}=2200$ being about $25-30 \%$ longer than the one at $\operatorname{Re}=2100$. 
As seen in figure 2 (left), at $\mathrm{Re}=2300$ the puff - the isolated turbulent patch featured as a dark segment at given time $t$ - splits irregularly, a first time at $t \approx 4700$ and a second time at $t \approx 7500$. The newborn puffs are separated from their parents by significant laminar gaps. A third splitting takes place from of the parent puff at $t \approx 8300$, but the corresponding child puff is hardly observable on the left border of the graph, i.e. for $\left(z-u_{\mathrm{m}} t\right) \simeq 0$. The result of this third splitting is better visible in figure 3 which displays the final state at $t=9000$. Here the azimuthally-unfolded streamwise component of the velocity close to the wall is displayed. This more readable representation was chosen after scrutinizing the velocity field at various distances from the centerline. The value $r=0.8$ offered the clearest discrimination and will be kept in forthcoming figures. The laminar gaps between successive puffs are less masked by the elongated but weak perturbations of the velocity field along the centerline, as can be understood from the consideration of figure 1.

At larger Re (figure 2, right), the turbulent region expands quite regularly. The whole region between the most upstream front and the leading edge is rather messy and can definitely not be considered as a train of pseudo-equilibrium puffs, though laminar intervals of significant length can still be transiently detected.

For all the values of Re that we have studied, the speed of the most upstream laminar-turbulent front is well defined. In the case of pseudo-equilibrium puffs at $\operatorname{Re}=2100$ we measured a trailing edge speed $u_{\text {te }} \approx 0.485$, which is slightly smaller than the bulk speed $u_{\mathrm{m}}=0.5$, as expected; see for example Hof et al. (2005) who display the relative trailing and leading edge speeds $u_{\mathrm{te}} / u_{\mathrm{m}}$ and $u_{\mathrm{le}} / u_{\mathrm{m}}$ in their Fig. 4. As soon as the splitting sets in, turbulence expands due to a gentle decrease of the trailing edge speed, $u_{\mathrm{te}}=0.463,0.454$, and 0.444 , for $\mathrm{Re}=2300,2400$, and 2500 respectively, while the leading edge speed $u_{\mathrm{le}}$ remains close to 1 and increases slowly. We measure $u_{\mathrm{le}}=0.494$ and 0.505 for $\mathrm{Re}=2400$ and 2500 , respectively, but no reliable value could be extracted for $\mathrm{Re}=2300$, due to the discrete distribution of splitting events. Returning to the case $\operatorname{Re}=2300$, three trailing edges corresponding to the three puffs can be identified near the end of the simulation for $t \gtrsim 7500$, the parent puff being separated from its children by a substantial laminar interval, while the grand-child is still somewhat locked to the child. Measured speeds from upstream to downstream are $u_{\mathrm{te}}=0.463,0.486$ and 0.493 , in qualitative agreement with results displayed in Avila et al. (2011), Fig. 1C. This confirms that the amount and quality of the laminar flow upstream of a puff in a given train, i.e. the distance between puffs, plays an important role in fixing its speed (Samanta et al 2011).

In all the cases considered, the final simulation was fixed by the condition that the leading edge of the puff or of the cluster of puffs remains sufficiently far from its own trailing edge, when taking periodic boundary conditions into account (see figure 2). We can thus safely consider the numerical domain as sufficiently elongated for the perturbations to be regarded as evolving along an infinitely long pipe.

Let us now illustrate the processes through which puffs split. Figures 4 and 5 display the first and second splitting events observed at $t \approx 4750$ and $t \approx 7100$ for 

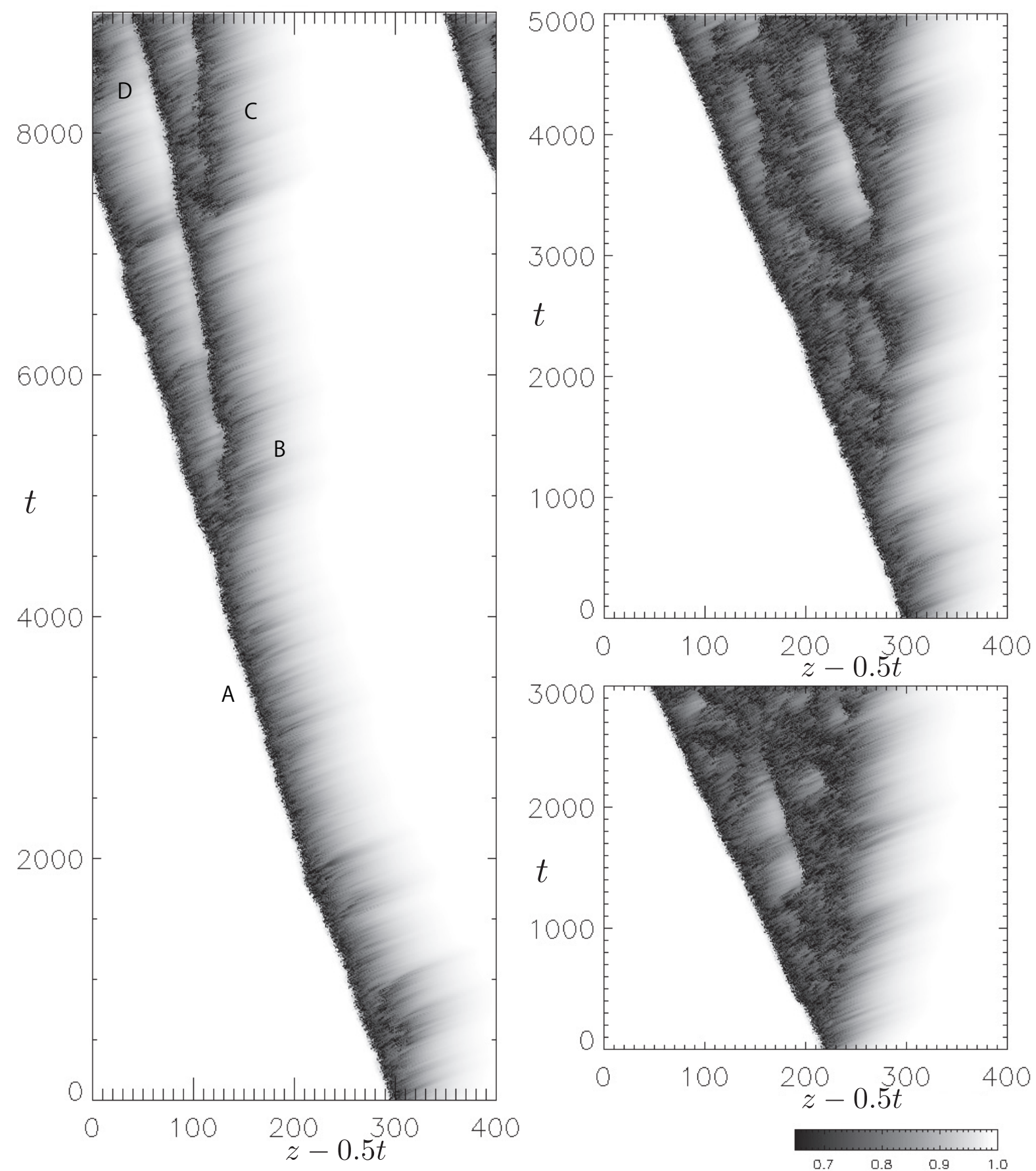

Figure 2. Spreading of turbulence for $R e=2300$ (left), $R e=2400$ (top-right), and $R e=2500$ (bottom-right). Spatiotemporal diagrams of the centerline streamwise velocity component are displayed in a reference frame moving at the mean velocity $u_{\mathrm{m}}=0.5$. White corresponds to laminar flow $u_{\mathrm{cl}}=1$, shades of gray to disturbed flow with lower values inside the puffs. 


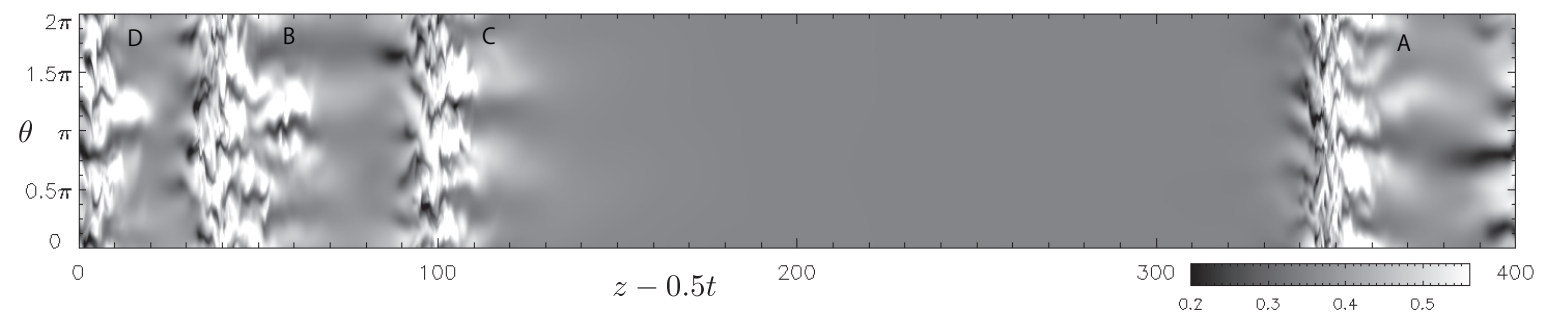

Figure 3. State reached at $t=9000$ for $\mathrm{Re}=2300$ showing four puffs. The streamwise component of the velocity at distance $r=0.8$, i.e. close to the wall, is represented in shades of gray in the $(\theta, z)$ plane, with the angle $\theta \in[0,2 \pi]$ along the vertical axis as a function of the axial coordinate $z \in[0,400]$ along the horizontal axis.

Re $=2300$ using the same representation as in figure 3. In both cases, before splitting, several streaks, alternately rapid and slow, can be observed at the leading edge. The second splitting, to be examined first (figure 5), shows a more intense pair protruding into the laminar region ahead $(t=7200,7240,7280)$ for $\theta \approx \pi / 2$. The disturbance then rapidly spreads azimuthally $(t=7320,7360)$. Turbulence next begins to collapse just upstream of this new puff head $(t=7400)$, progressively forming a clear trailing edge (visible from $t=7600 \mathrm{on}$ ). This description is consistent with former experimental observations (Lindgren 1959, Avila et al 2011). In fact the second puff (B in figure $2-5)$ continues to emit streak structures ahead of itself $(t=7880)$, that tend to fill the laminar gap $(t=7960)$. A sizable permanent laminar gap between the two puffs (B and $\mathrm{C}$ ) is then present for $t \geq 8080$ and persists later as testified by the snapshot at $t=9000$ displayed in figure 3 .

Figure 6 gives an illustration of the local intensity of the self-sustainment process (Waleffe 1997) zoomed on the region of splitting. It displays iso-surfaces of the fluctuation component $u_{z}-\left\langle u_{z}\right\rangle_{\theta z}=-0.1$ (gray) and $\omega_{\theta}-\left\langle\omega_{\theta}\right\rangle_{\theta z}=1.5$ (black). The former locate the slow speed streaks and the latter the high intensity spanwise vortices. The top and middle frames, at $t=7200$ and 7280 illustrate an intense streaky pattern induced by lift-up and its subsequent breakdown around $\theta=2 \pi / 3$ and $z=100$. The bottom frame, at a later time, $t=7400$, shows that the apparently laminar space generated by the on-going splitting is filled with diametrically opposed streaky structures around $\theta=0, \pi$ ahead of $z-u_{\mathrm{m}} t \approx 92$ forming the leading edge of the upstream puff, while the trailing edge of the downstream puff is not yet well formed.

Let us now focus on the first splitting event, around $t \approx 4750$ and $z-u_{\mathrm{m}} t \approx 100$ (figure 4). Again at start $(t=4600)$ an azimuthally periodic streaky disturbance with wavenumber $\approx 3$ is observed. The parent puff now emits two diametrically opposite strong low-speed streaks $(t=4720,4760)$ that, not surprisingly, quickly contaminate the full perimeter of the pipe $(t=4800)$. However, the collapse of turbulence allowing for the identification of two separate puffs seems more difficult to achieve. Premises of a laminar gap between parent and child are visible at $t=4920$ but they are well separated only for $t \geq 5000$. This gap is never observed to close (see $t=5200$ ) but instead progressively widens due to the velocity difference between the two different 

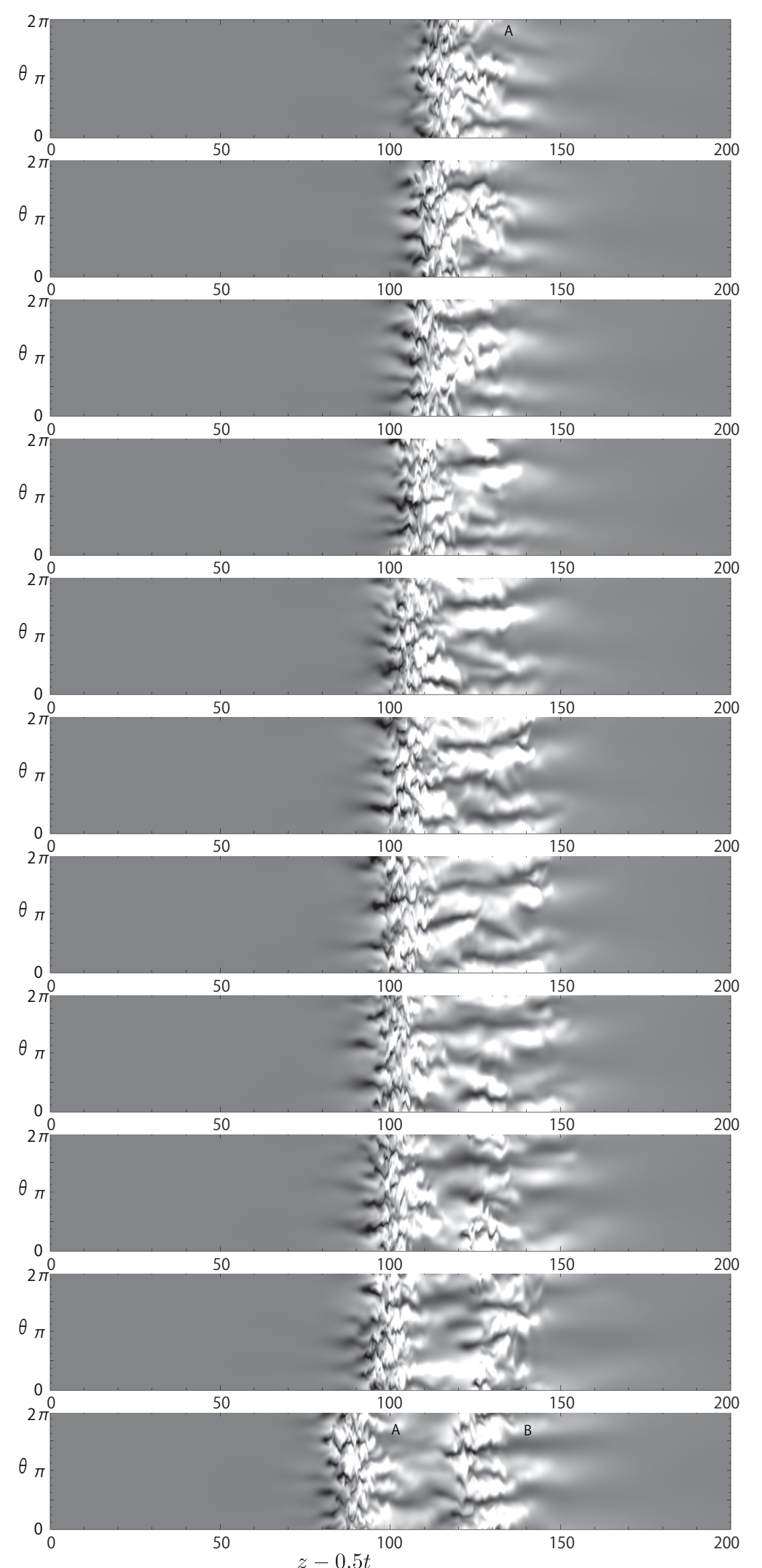

Figure 4. Snapshots taken during the first puff splitting observed for $\operatorname{Re}=2300$. Same representation as in figure 3. From top to bottom: $t=4600,4640,4680,4720$, 4760, 4800, 4840, 4880, 4920, 5000, 5200. 

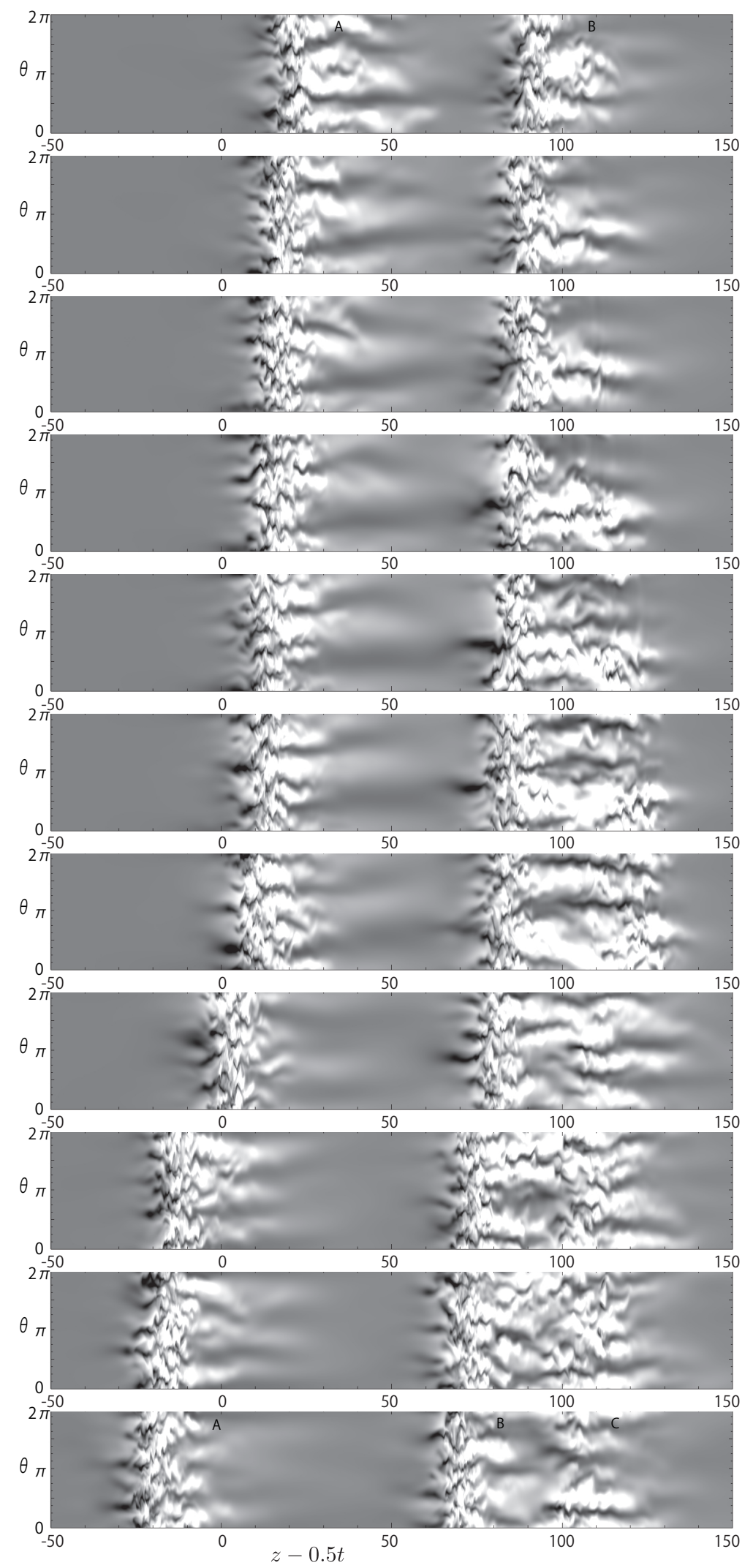

Figure 5. Snapshots taken during the second puff splitting observed for $\operatorname{Re}=2300$. Same representation as in figure 3. From top to bottom, $t=7120,7200,7240,7280$, $7320,7360,7400,7600,7880,7960,8080$. Periodic boundary conditions have been used to display the solution in the most appropriate window (compare with figure 3 ). 

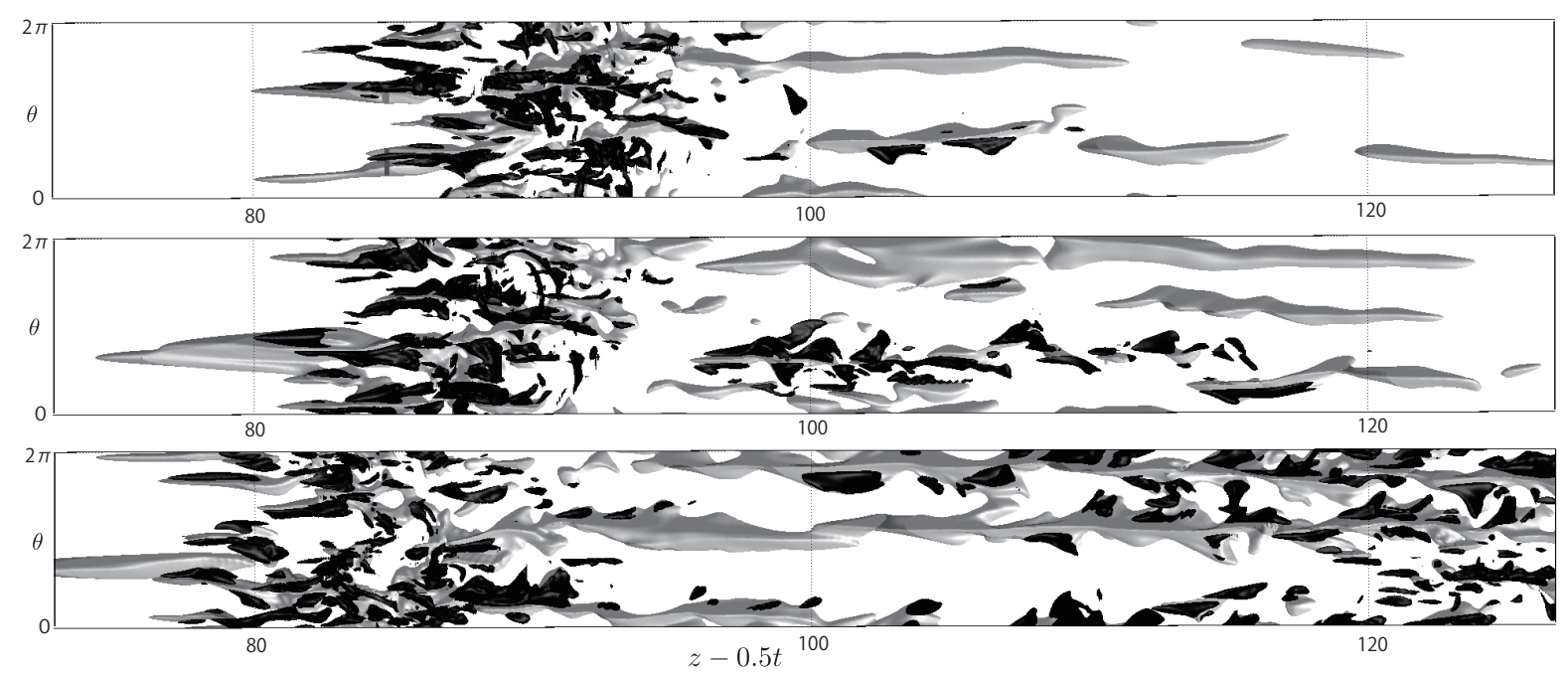

Figure 6. Disturbances during the second puff splitting observed for $\operatorname{Re}=2300$. Inward view from the pipe wall in cylindrical coordinate. Gray and black iso-surfaces represent $u_{z}-\left\langle u_{z}\right\rangle_{\theta z}=-0.1$ and $\omega_{\theta}-\left\langle\omega_{\theta}\right\rangle_{\theta z}=1.5$, respectively. From top to bottom, $t=7200,7280,7400$.

trailing edge speeds (see also figure 2, left).

Turning to larger Re, here Re $=2400$ (figure 7), it is clear that "puff splitting" has a little changed its meaning. Downstream of the leading edge, one can still recognize the emission of streaky protuberances and their possible failing at nucleating a puff (see the pair of streaks at $t=710$ and $\pi<\theta<3 \pi / 2$ that collapses around $t=910$, and also consider figure 2, top-right for $700<t<1000$ where the widening and next narrowing of the puff is clearly visible). This aborted event results in a conspicuous temporal fluctuation of the length of the puff. When the azimuthal expansion of the protuberance is successful and turbulence spreads downstream, there may also be no opening of a gap behind the leading edge, yielding a compact anomalously elongated puff that can last for a substantial amount of time $(1180 \lesssim t \lesssim 1420)$. Such longer puffs appear however to be unstable, and turbulence can collapse locally, somewhere strictly between the leading edge and the trailing edge (Avila and Hof 2013). An example of collapse close to the leading edge is given for $3150 \lesssim t \lesssim 3350$. Another one close to the trailing edge can be detected at $t \approx 3700$ in figure 2 , top-right, yielding a separate puff at the rear of the turbulent domain. The last example is a general collapse of turbulence all over the pipe's cross-section over a significant downstream length, observed near the end of our simulation $(t=4900,5000)$.

This limited set of illustrations cannot claim to give a complete catalogue of the processes observable in the upper transitional range, where the probability of puff splitting is no longer negligible and the puffs make their way to becoming "slugs." Let us notice already at this stage that macroscopic features of the flow pattern at the scale of the puff itself (turbulence breakdown, flow reorganization within the laminar gap) seem to result from the combination/interplay of microscopic high-frequency processes 
at the scale of the local streak structures (emission of streaky protuberances, azimuthal expansion), which we discuss further in the next section.

\section{Discussion}

In this paper, by means of well-resolved but computationally demanding numerical simulations extending previous work (Shimizu and Kida 2009), we have investigated the part of the transitional range of pipe flow where turbulence is sustained with essentially full probability, $\operatorname{Re}>\operatorname{Re}_{\mathrm{g}}$ (Avila et al 2011), while isolated gentle pseudo-equilibrium puffs change into expanding trains of puffs (Nishi et al 2008) before turning into fastergrowing slugs (Duguet et al 2010).

At the lowest end of the transitional range $\left(R e<R_{g}\right)$ the much studied decay of puffs - see (Hof et al 2008) and references therein - has been interpreted using the familiar concept of transient chaos within the framework of deterministic dynamical systems theory (Eckhardt et al 2008, Avila et al 2013). This approach straightforwardly explains the exponentially decreasing lifetime distributions observed experimentally and happens to be quite acceptable only well below $\operatorname{Re}_{\mathrm{g}}$ where puffs are short and, spatially speaking, relatively coherent.

However, as we have reported, the area occupied by turbulent fluctuations statistically elongate when Re increases. Spatial coherence supporting the dynamical systems approach then becomes questionable. A concurrent interpretation of the decay in terms of extreme events (Clusel and Bertin 2008) has indeed been proposed (Goldenfeld et al 2010), able to account for both the distribution of lifetimes and the variation of their means with Re. A similar approach has been put forward to explain the decay of turbulent bands in PCF below its own global stability threshold (Manneville 2011, Faranda et al 2014) and further adapted to deal with the growth of the oblique laminar-turbulent pattern conspicuous in its transitional range (Manneville 2012) and to which the puff splitting regime closely corresponds.

The general context is that of spatiotemporal dynamics appropriate to extended systems (Pomeau 1986) with two facets: $(i)$ at the local scale, a growth-decay problem treated as a manifestation of spatiotemporal intermittency (Chaté and Manneville 1995), a deterministic but spatiotemporally chaotic variant of a fully stochastic process named directed percolation (Hinrichsen 2000), and (ii) at the global scale, a nucleation problem (Bergé et al. 1998) as encountered in the theory of first-order phase transitions in thermodynamics, viz. the liquid-gas transition. The first facet was shown to be relevant to the transition in pipe flow, at least in the model discussed in (Avila et al 2011, Barkley 2011b); see also (Sipos and Goldenfeld 2011). Large deviations (Clusel and Bertin 2008) are directly connected to the second facet: Breakdown of turbulence over large enough turbulent segment, be it a long puff (Goldenfeld et al 2010) or a portion of oblique turbulent band as in Couette flow, is indeed easily understood as resulting from the spontaneous nucleation of critical laminar trough (Manneville 2011, Faranda et al 2014). The growth problem of interest here fits this framework well, in much the same 

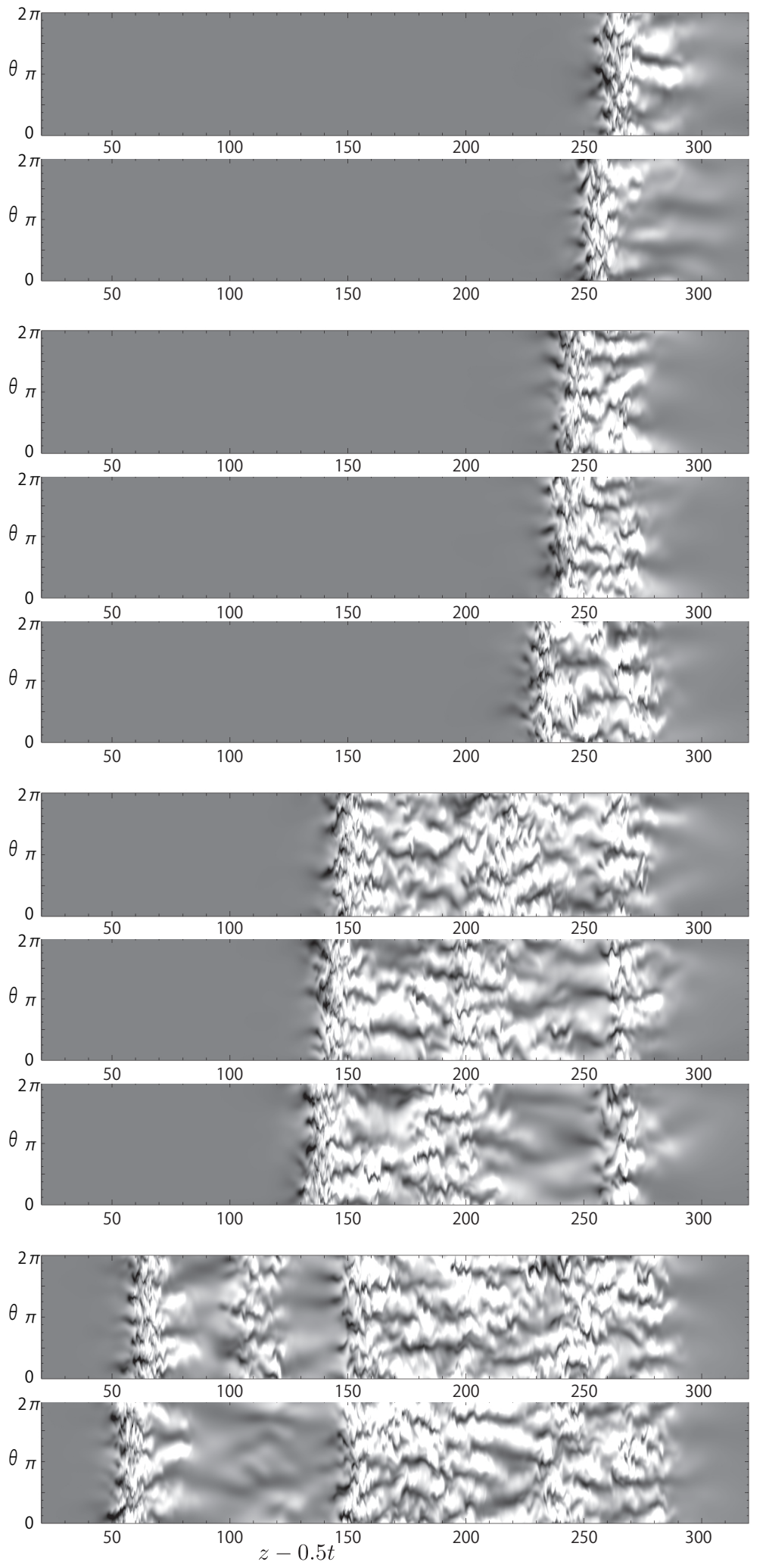

Figure 7. Snapshots of different cases observed for $\mathrm{Re}=2400$. Same representation as in figure 3. From top to bottom, $t=710,910$ (aborted splitting), $t=1180,1240$, 1420 (extension without separation), $t=3150,3250,3350$ (separation of leading puff), $t=4900,5000$ (breakdown of last but one puff). 
way as in $\mathrm{PCF}$ for which probabilities introduced in the directed percolation approach have been computed (Duguet et al 2011). Here, from the fine oblique lines displayed in the spatiotemporal diagrams of figure 2 and corresponding to the traces of the streaks emitted by the puff near the centerline, a dominant frequency of streak emission of about 0.1 per unit time, can be inferred, which corresponds to a period of $\approx 10 a / 2 U$. The order of magnitude of this period is comparable to that of invariant solutions relevant to the pipe (Duguet et al 2008, Avila et al 2013, Kawahara et al 2012), typical of "microscopic" processes alluded to above. Furthermore, the irregularly varying length and gray intensity of the obliques traces is a clear indication of the stochastic character of this process and quantitative support needs further scrutiny. The large-deviation consequences at the "macroscopic" scale of local processes at the scale of the streak thus seem a key element in order to account for the catalogue of events involved in puff expansion, as highlighted in the previous section. Morever, at the microscopic level, perturbations flushed downstream and potentially nucleating new puffs are advected with a mean velocity larger than that of the parent puff. It would thus be interesting to examine how large scale flows, that appear to play an important role in plane flows (Manneville 2012, Duguet and Schlatter 2013, Lemoult et al 2013), contribute to the splitting process in pipe flow.

At any rate, statistical physics (Pomeau 1986, Bergé et al. 1998) and its derivatives, non-equilibrium phase transitions (Hinrichsen 2000), large deviations and extreme values theory (Clusel and Bertin 2008), have provided thoughtful insights in the transition to turbulence of pipe flow and PCF and will certainly continue to enlighten the dynamics of transitional wall-bounded flows.

\section{Acknowledgments}

Part of this work has been performed within the framework of the EGIDE-PHC SAKURA project \#25714NH funded by the Japan Society for the Promotion of Science and the French Ministry of Foreign Affairs. The authors are grateful to the referees for their constructive remarks and YD would like to thank Prof. Avila for interesting discussions.

\section{References}

Avila K, Moxey D, de Lozar A, Avila M, Barkley D and Hof B 2011 The onset of turbulence in pipe flow Science 333, 192-196.

Avila M and Hof B 2013 Nature of laminar-turbulence intermittency in fluid flow Phys. Rev. E 87, 063012 .

Avila M, Mellibovsky F, Roland N and Hof B 2013 Streamwise-Localized Solution at the Onset of Turbulence in Pipe flow Phys. Rev. Lett. 110, 224502.

Bandyopadhyay P R 1986 Aspects of the equilibrium puff in transitional pipe flow J. Fluid Mech. 163, 439-458.

Barkley D 2011a Modeling the transition to turbulence in shear flows J. Physics: Conference Series 318, 032001. 
Barkley D $2011 b$ Simplifying the complexity of pipe flow Phys. Rev. E 84, 016309.

Bergé P, Vidal C and Pomeau Y 1998 L'espace chaotique (Paris: Hermann).

Chaté H and Manneville P 1995 Spatiotemporal intermittency NATO ASI series, Series B: Physics 341 (New York: Plenum Press).

Clusel M and Bertin E 2008 Global fluctuations in physical systems: a subtle interplay between sum and extreme value statistics Int. J. Mod. Phys. B 22, 3311-3368.

Coles D 1962 Mécanique de la Turbulenc pp. 229-248 (Paris: Éditions du CNRS).

Duguet Y, Le Maître O and Schlatter P 2011 Stochastic and deterministic motion of a laminar-turbulent front in a spanwisely extended Couette flow Phys. Rev. E 84, 066315.

Duguet Y, Pringle C and Kerswell R 2008 Relative periodic orbits in transitional pipe flow Phys. Fluids 20, 114102.

Duguet Y and Schlatter P 2013 Oblique laminar-turbulent interfaces in plane shear flows Phys. Rev. Lett. 110, 034502.

Duguet Y, Willis A and Kerswell R 2010 Slug genesis in cylindrical pipe flow J. Fluid Mech. 663, 180208.

Eckhardt B, Faisst H, Schmiegel A and Schneider T M 2008 Dynamical systems and the transition to turbulence in linearly stable shear flows Phil. Trans. R. Soc. A 366, 1297-1315.

Faranda D, Lucarini V, Manneville P and Wouters J 2014 On using extreme values to detect global stability thresholds in multi-stable systems: The case of transitional plane Couette flow Chaos, Solitons and Fractals in press.

Goldenfeld N, Guttenberg N and Gioia G 2010 Extreme fluctuations and the finite lifetime of the turbulent state Phys Rev. E 81, 035304.

Hinrichsen H 2000 Non-equilibrium critical phenomena and phase transitions into absorbing states Advances in Physics 49, 815-958.

Hof B, de Lozar A, Kuik D J and Westerweel J 2008 Repeller or attractor? Selecting the dynamical model for the onset of turbulence in pipe flow Phys. Rev. Lett. 101, 214501.

Holzner M, Song B, Avila M and Hof B 2013 A Lagrangian approach to the interface velocity of turbulent puffs in pipe flow J. Fluid Mech. 723, 140-162.

Kawahara G, Uhlmann M and van Veen L 2012 The significance of simple invariant solutions in turbulent flows Annu. Rev. Fluid Mech. 44, 203-225.

Lemoult G, Aider J L and Wesfreid J 2013 Turbulent spots in a channel: large-scale flow and selfsustainability J. Fluid Mech. 731, R1-12.

Lindgren R 1959 Liquid flow in tubes II. The transition process under less disturbed inlet flwo conditions Arkiv fur Fysik 15, 503-519.

Manneville P 2011 On the decay of turbulence in plane Couette flow Fluid Dyn. Res. 43, 065501.

Manneville P 2012 On the growth of laminar-turbulent patterns in plane Couette flow Fluid Dyn. Res. 44, 031412 .

Manneville P and Rolland J 2011 On modelling transitional turbulent flows using under-resolveddirect numerical simulations: the case of plane Couette flow Theor. Comput. Fluid Dyn. 25, 407-420

Moxey D and Barkley D 2010 Distinct large-scale turbulent-laminar states in transitional pipe flow Proc. Nat. Acad. Sci. 107, 8091-8096.

Nishi M, Ünsal B, Durst F and Biswas G 2008 Laminar-to-turbulent transition of pipe flows through puffs and slugs J. Fluid Mech. 614, 425.

Pomeau Y 1986 Front motion, metastability and subcritical bifurcations in hydrodynamics Physica D: Nonlinear Phenomena 23, 3-11.

Reynolds O 1883 An experimental investigation of the circumstances which determine whether the motion of water shall be direct or sinuous, and of the law of resistance in parallel channels Proc. R. Soc. London 35, 84-99.

Romanov V 1973 Stability of plane-parallel Couette flow Functional Analysis and Its Applications $\mathbf{7}(2), 137-146$.

Rotta J 1956 Experimental contributions to the development of turbulent flow ina pipe Ing. Arch. 
24, 258.

Salwen H, Cotton F W and Grosch C E 1980 Linear stability of Poiseuille flow in a circular pipe $J$. Fluid Mech. 98, 273-284.

Samanta K, de Lozar A and Hof B 2011 Experimental investigation of laminar turbulent intermittency in pipe flow J. Fluid Mech. 681, 193-204.

Shimizu M and Kida S 2008 Structure of a turbulent puff in pipe flow J. Phys. Soc. Japan 77, 4401.

Shimizu M and Kida S 2009 A driving mechanism of a turbulent puff in pipe flow Fluid Dyn. Res. 41, 045501.

Sipos M and Goldenfeld N 2011 Directed percolation describes lifetime and growth of turbulent puffs and slugs Phys. Rev. E 84, 035304.

Sreenivasan K R and Ramshankar R 1986 Transition intermittency in open flows, and intermittency routes to chaos Physica D 23, 246-253.

Waleffe F 1997 On a self-sustaining process in shear flows Phys. Fluids 9, 883-900.

Wygnanski I and Champagne F 1973 On transition in a pipe. Part 1. The origin of puffs and slugs and the flow in a turbulent slug J. Fluid Mech. 59, 281-335.

Wygnanski I, Sokolov M and Friedman D 1975 On transition in a pipe. Part 2. The equilibrium puff J. Fluid Mech. 69, 283-304. 